\title{
Tétanos postcirconcision : à propos de 16 cas colligés à la Clinique des maladies infectieuses du CHNU de Fann (Dakar)
}

\section{Post-circumcision Tetanus: Epidemiological, Clinical, Prognostic and Evolutionary Aspects of 16 Cases Collected at the Department of Infectious and Tropical Diseases in Fann Hospital (Dakar)}

\author{
K. Diallo Mbaye $\cdot$ N.A. Lakhe $\cdot$ K. Sylla $\cdot$ V.M.P. Cissé Diallo $\cdot$ A. Massaly $\cdot$ D. Ka $\cdot$ N.M. Fall $\cdot$ \\ L. Fortes Déguénonvo - C.T. Ndour - M. Soumaré - M. Seydi
}

Reçu le 30 juillet 2018 ; accepté le 19 décembre 2018

C Société de pathologie exotique et Lavoisier SAS 2019

Résumé Cette étude rétrospective descriptive, réalisée à la Clinique des maladies infectieuses du CHNU de Fann du $1^{\text {er }}$ janvier 2012 au 31 décembre 2016 avait pour but de décrire les aspects épidémiologiques et évolutifs du tétanos postcirconcision et de formuler des recommandations en termes de prévention. Durant la période d'étude, 16 cas de tétanos postcirconcision ont été colligés sur un total de 452 patients atteints de tétanos, soit une fréquence hospitalière de 3,5\%. L'âge médian était de huit ans (3-40). Aucun des patients ne disposait de carnet de vaccination. Dans $63 \%$ des cas, la circoncision a été effectuée à domicile, par un praticien dont la qualification n'a pas été documentée dans $63 \%$ des cas. Tous les signes du tétanos ont été retrouvés avec le trismus ( $100 \%)$, la dysphagie (63\%) et les paroxysmes $(88 \%)$. Il s'agissait surtout de formes de moyenne gravité classées au stade II de Mollaret dans 14 cas, et au score 3 de Dakar dans sept cas. La durée médiane d'hospitalisation était de dix jours [6-15]. Des complications mécaniques ou infectieuses ont été notées dans deux cas, avec une létalité de $13 \%$. Le tétanos postcirconcision est une réalité au Sénégal. Une bonne sensibilisation de la communauté, la formation continue du personnel et la mise en place de stratégies de rattrapage vaccinal des enfants en âge d'être circoncis devraient permettre d'éradiquer cette forme de tétanos.

K. Diallo Mbaye $(\varangle) \cdot$ N.A. Lakhe $\cdot$ V.M.P. Cissé Diallo

A. Massaly $\cdot$ D. Ka $\cdot$ N.M. Fall - L. Fortes Déguénonvo ·

C.T. Ndour $\cdot$ M. Soumaré $\cdot$ M. Seydi

Clinique des maladies infectieuses,

CHNU de Fann, BP 5035 Dakar, Sénégal

e-mail : diallokhardiata@gmail.com

K. Sylla

Laboratoire de parasitologie-mycologie, faculté de médecine, université Cheikh-Anta-Diop de Dakar, Sénégal
Mots clés Circoncision · Tétanos · Hôpital · Fann · Dakar · Sénégal $\cdot$ Afrique intertropicale

Abstract This retrospective, descriptive study carried out in the Infectious Diseases Department of CHNU, Fann from $1^{\text {st }}$ January 2012 to 3 st December 2016 aimed to describe the epidemiological aspects and the course of postcircumcision tetanus and to formulate public health recommendations. During our study period, 16 cases of postcircumcision tetanus were collected out of a total of 452 patients with tetanus, equivalent to a rate of $3.5 \%$. The median age was 8 years (3-40). None of the patients had a vaccination card. In $63 \%$ of cases, circumcision was performed at home, by a practitioner whose qualification was not documented in $63 \%$ of cases. All signs of tetanus were found with trismus $(100 \%)$, dysphagia $(63 \%)$ and paroxysms $(88 \%)$. These were mostly Mollaret stage II forms in 14 cases, and grade 3 Dakar score in 7 cases. The median duration of hospitalization was 10 days [6-15]. Mechanical or infectious complications were noted in two cases, with a lethality rate of $13 \%$. Post-circumcision tetanus is a reality in Senegal. Good community awareness, continuous training of staff and implementation of vaccination catch-up strategies for children of circumcised age should eradicate this form of tetanus.

Keywords Circumcision - Tetanus · Hospital · Fann · Dakar · Sénégal · Sub-Saharan Africa

\section{Introduction}

La circoncision masculine est l'une des procédures chirurgicales les plus courantes dans le monde. Selon des études récentes [1,2], elle protège les individus contre le VIH, mais 
consiste surtout à perpétuer des convictions religieuses dans l'islam et le judaïsme, alors que dans les cultures africaines elle était associée à des rites de passage à l'âge adulte.

Cette pratique peut être à l'origine de complications graves, notamment des mutilations [5], des cas de tétanos postcirconcision.

Cette étude réalisée à la Clinique des maladies infectieuses du CHNU de Fann à Dakar se fixait pour objectifs de déterminer la place du tétanos secondaire à une circoncision, d'en décrire les aspects épidémiologiques, cliniques et évolutifs, et de formuler des recommandations en termes de santé publique.

\section{Patients et méthodes}

Il s'agissait d'une étude rétrospective, descriptive à partir des dossiers médicaux de patients hospitalisés dans le service des maladies infectieuses du CHNU de Fann du $1^{\text {er }}$ janvier 2012 au 31 décembre 2016. Le diagnostic du tétanos postcirconcision a été retenu en l'absence de toute autre porte d'entrée potentielle. Pour chaque malade répondant aux critères d'inclusion, les données suivantes ont été recueillies : l'âge, le sexe, le statut vaccinal, la qualification du circonciseur, le lieu de la circoncision, les manifestations cliniques (trismus, contractures, dysphagie, paroxysmes), le stade et le score de gravité, et l'évolution. Tous les patients ont reçu le traitement standard du tétanos comprenant le parage de la porte d'entrée, la sérothérapie hétérologue par voie sousoccipitale, une antibiothérapie à base de métronidazole et un traitement symptomatique à base de sédatifs.

La saisie et l'analyse des données ont été faites grâce au logiciel Epi-Info ${ }^{\mathrm{TM}} /$ Epi-Data version 6.04 (CCDC-OMS). Les variables quantitatives ont été exprimées en médianes et en valeurs extrêmes, et les variables qualitatives sous forme de proportions.

\section{Résultats}

Durant les cinq ans de la période d'étude, 452 patients ont été admis dans le service pour tétanos, sur un total de 5032 admissions, toutes pathologies confondues, soit un taux de morbidité proportionnelle de $8,9 \%$. Sur les 452 cas de tétanos, 16 étaient secondaires à une circoncision, soit une fréquence hospitalière de 3,5\%.

Le nombre de cas variait en fonction des années, avec une médiane de trois cas par an et un maximum de neuf cas en 2015. L'âge médian était de huit ans (3-40). Les deux tiers des cas provenaient des zones suburbaines. Dans $63 \%$ des cas, la circoncision a eu lieu à domicile, alors que la qualification du circonciseur n'a été précisée que dans moins de la moitié des cas. Aucun de ces patients ne disposait de carnet de vaccination.

Sur le plan clinique, la durée médiane d'incubation était de 11 jours (10-14), celle d'invasion était de deux jours (1-3).

Tous les patients ont présenté une forme aiguë généralisée. Le trismus et les contractures étaient présents chez tous les patients et les paroxysmes dans 14 cas sur 16. Les principales manifestations cliniques observées à l'admission ont été rapportées sur la figure 1.

Sur le plan pronostique, le stade II de la classification de Mollaret était largement le plus représenté avec 14 cas, tandis que le score de Dakar était égal à 3 dans sept cas. La répartition des patients selon les classifications à visée pronostique par stade de Mollaret et par score de Dakar a été rapportée dans le tableau 1. La durée médiane d'hospitalisation était de dix jours (7-15). L'évolution a été favorable, sans séquelles, chez 14 patients, mais il y a eu deux décès, soit un taux de létalité de $13 \%$.

\section{Discussion}

Durant la période d'étude de cinq ans, 16 cas de tétanos postcirconcision ont été recensés parmi 452 cas, toutes portes d'entrée confondues, avec une médiane de trois cas par an, soit une diminution de moitié par rapport aux données de 2008 dans le même service [3]. Cette fréquence est certes faible $(3,5 \%)$, mais doit être considérée comme préoccupante pour une affection qui ne devrait plus exister.

Conformément aux données de la littérature en contexte africain $[3,4]$, aucun de ces patients n'avait été correctement vacciné contre le tétanos, avec documentation dans un carnet de vaccination. Le manque d'éducation et le bas niveau de vie des populations sont les principaux déterminants du

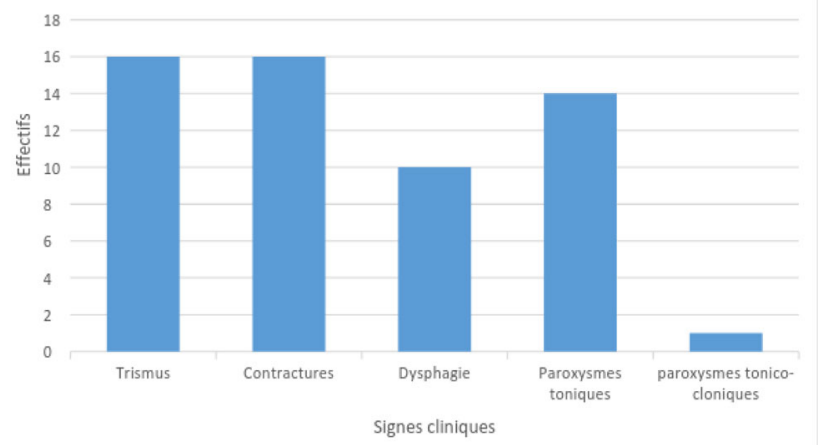

Fig. 1 Répartition des cas de tétanos postcirconcision selon les signes cliniques à l'admission à la Clinique des maladies infectieuses du CHNU Fann, 2012-2016 / Distribution of postcircumcision tetanus cases according to clinical signs at admission at the Department of Infectious Diseases, Fann Hospital 2012-2016 
Tableau 1 Répartition des cas de tétanos postcirconcision à la Clinique des maladies infectieuses CHNU Fann 20122016 selon la gravité par stade de Mollaret et par score de Dakar / Distribution of post-circumcision tetanus cases by severity by Mollaret stage and Dakar score at the Department of Infectious and Tropical Diseases, Fann Hospital 2012-2016

\begin{tabular}{|ll|}
\hline Stade & Effectif \\
\hline Stade I & 1 \\
II & 14 \\
III & 1 \\
Total & 16 \\
\hline Score & Effectif \\
\hline 1 & 4 \\
2 & 5 \\
3 & 7 \\
Total & 16 \\
\hline
\end{tabular}

statut vaccinal. Le fait que $63 \%$ de ces patients provenaient de zones suburbaines, notamment de la banlieue de Dakar caractérisée par la surpopulation, l'enclavement, le bas niveau socio-économique et la difficulté d'accès aux structures de soins, pourrait expliquer en partie ce constat. L'âge médian était de huit ans avec une prédominance de la tranche d'âge cinq à dix ans. Ce constat est loin d'être surprenant au Sénégal, où la circoncision est réalisée au cours des premières années de vie, le plus souvent avant l'adolescence. À cet âge, la série de vaccinations du Programme élargi de vaccination est théoriquement complète, et pour ceux qui ont eu la chance de le recevoir, il convient de respecter les différentes doses de la vaccination, sans lesquelles le risque de tétanos redevient important. La mise en place de sessions de rattrapage au profit des enfants en âge d'être circoncis devrait aider à immuniser le maximum d'entre eux et donc à réduire de manière drastique les cas de tétanos postcirconcision.

La circoncision a été réalisée à domicile dans deux tiers des cas, constat régulièrement fait aussi bien au Sénégal que dans la sous-région et qui pose un véritable problème médicolégal, d'autant que, dans la majorité des cas, l'intervention est réalisée par un acteur de santé, le plus souvent un infirmier. Plusieurs facteurs peuvent expliquer la persistance de ces pratiques, dont la pauvreté, l'inaccessibilité des soins, le bas niveau d'instruction et la difficulté de lutter contre des pratiques traditionnelles fortement ancrées dans les sociétés africaines [3]. La médicalisation effective de cette pratique traditionnelle et une bonne formation des acteurs de santé sur l'importance de la vaccination et de la possession d'un carnet de vaccination à jour devraient permettre une nette amélioration de la situation.
Sur le plan clinique, la forme aiguë généralisée a été la règle. La fréquence élevée de cette forme généralisée quelle que soit la porte d'entrée a été rapportée par plusieurs auteurs $[3,4]$. Elle s'explique surtout par le diagnostic tardif du tétanos lié en partie à un itinéraire thérapeutique complexe.

En cours d'évolution, deux patients ont présenté au moins une complication dont l'un de nature mécanique avec spasmes laryngés et l'autre de nature infectieuse, soit un taux de loin inférieur à la série de Soumaré et al. qui avaient rapporté $24 \%$ de complications [4].

Au plan pronostique, il s'agissait toutefois de formes classées aux stades I ou II de Mollaret à l'hospitalisation dans $94 \%$ des cas, de moindre gravité.

On comprend dès lors le faible taux de létalité noté dans notre série, cependant plus élevé que les $7 \%$ rapportés par Soumaré et al.

\section{Conclusion}

Le tétanos postcirconcision, un tétanos du jeune enfant de gravité moyenne, est encore une réalité au Sénégal, malgré l'existence d'un vaccin efficace et accessible.

Une bonne communication envers la communauté sur l'importance de la vaccination des enfants et la possession d'un carnet de vaccination, associée à la formation du personnel médical sur la médicalisation effective de la circoncision, sont les gages du succès dans la lutte contre cette forme de tétanos.

Liens d'intérêts : Les auteurs déclarent ne pas avoir de liens d'intérêts.

\section{Références}

1. Kibira SP, Sandøy IF, Daniel M, et al (2016) A comparison of sexual risk behaviours and HIV seroprevalence among circumcised and uncircumcised men before and after implementation of the safe male circumcision programme in Uganda. BMC Public Health 16:7. doi: 10.1186/s12889-015-2668-3

2. Mavundla TR, Netswera FG, Bottoman B, Toth F (2009) Rationalization of indigenous male circumcision as a sacred religious custom: health beliefs of Xhosa men in South Africa. J Transcult Nurs 20:395-404. doi: 10.1177/1043659609340801. Epub 2009 Jul 8

3. Soumaré M, Seydi M, Dia NM, et al (2008) Le tétanos postcirconcision à Dakar, Sénégal. Bull Soc Pathol Exot 101:54-57 [http://www.pathexo.fr/documents/articles-bull/T101-1-3077-4p.pdf]

4. Soumaré M, Seydi M, Ndour CT, et al (2005) Aspects épidémiologiques, cliniques et pronostiques du tétanos juvénile à Dakar. Bull Soc Pathol Exot 98:371-3 [http://www.pathexo.fr/documents/articles-bull/T98-5-2729-3p.pdf]

5. Sylla C, Diao B, Diallo AB, et al (2003) Les complications de la circoncision. À propos de 63 cas. Prog Urol 13:266-72 